\title{
Review
}

\section{The definition of tuberculosis infection based on the spectrum of tuberculosis disease}

Latent tuberculosis infection was the term traditionally used to indicate tuberculosis (TB) infection. This term was used to define "a state of persistent immune response to stimulation by Mycobacterium tuberculosis antigens through tests such as the tuberculin skin test (TST) or an interferon- $\gamma$ release assay (IGRA) without clinically active TB". Recent evidence indicates that the spectrum from TB infection to TB disease is much more complex, including a "continuum" of situations didactically reported as uninfected individual, TB infection, incipient TB, subclinical TB without signs/symptoms, subclinical TB with unrecognised signs/symptoms, and TB disease with signs/symptoms. Recent evidence suggests that subclinical TB is responsible for important $M$. tuberculosis transmission. This review describes the different stages described above and their relationships. It also summarises the new developments in prevention, diagnosis and treatment of TB infection as well as their public health and policy implications.

\section{Educational aims}

- To describe the evolution of the definition of "tuberculosis infection" and didactically describe the continuum of stages existing between TB infection and disease.

- To discuss the recommended approaches to prevent, diagnose and treat TB infection.

\section{Introduction}

The World Health Organization (WHO) estimated that in $2019 \sim 10$ million individuals were affected by tuberculosis (TB), with 1.4 million dying from the disease, and that a quarter of the global population was infected by Mycobacterium tuberculosis (Mtb) [1].

The evolution from TB infection to disease is now described as a "continuum process". Understanding of this is important to appreciate what is new on prevention, diagnosis and treatment of TB infection. https://bit.ly/3jauRKA

Cite as: Migliori GB, Ong CWM, Petrone L, et al. The definition of tuberculosis infection based on the spectrum of tuberculosis disease. Breathe 2021; 17 : 210079 


\section{TB transmission and pathogenesis}

Mtb is the causative agent of TB. Historically the diagnosis of TB was performed taking advantage of the alcohol acid-fast properties of the bacillus. The direct sputum smear examination, developed by Robert Koch, is still largely used after more than one century [2, 3], to complement the diagnostic armamentarium that includes TB culture and a new generation of rapid nucleic acid amplification tests.

TB is an airborne-transmitted disease: the bacilli are transmitted by inhaling droplet nuclei of dimensions ranging from 1 to $5 \mu \mathrm{m}$ from contagious individuals coughing (but also speaking, singing and sneezing) [4, 5]. Although in the majority of cases Mtb clearance takes place, infection may occur, either leading to a dormant state of the bacilli, or progressing to TB disease $[5,6]$.

Following exposure to Mtb, an estimated 20-25\% of individuals are infected, among whom $5-10 \%$ can develop active disease within the first 5 years $[7,8]$. In the remaining $90 \%$ of TB-infected individuals, the immune system, through innate and adaptive responses, controls the replication of the pathogen. In about $10 \%$ of the individuals, TB infection can evolve to TB disease during their lifetime. Among the different risk/predisposing factors described, we mention co-infection with HIV and other pathogens, including severe acute respiratory syndrome coronavirus 2 (SARS-CoV-2) [9-11], and comorbidities like diabetes, genetic predisposing factors, malnutrition, treatment with glucocorticoids or biological agents, solid organ transplantation, and malignancies [5].

Evidence indicates that in individuals co-infected with HIV the probability of developing TB disease given infection is high, up to $10 \%$ per year and $50 \%$ during life. Much less evidence is available for coronavirus disease 2019 (COVID-19). Several studies demonstrated that active TB associates with both good and severe COVID-19 outcomes [12-16] and it has been postulated that both diseases may impact one another. Co-infected individuals fail in building a SARS-CoV-2 immune response and show a skewed functional profile of the SARSCoV-2 specific T-cells [17]. Moreover, the COVID-19 lymphopenia and the steroid therapy during COVID19 in subjects with TB infection may increase the risk to progress to active TB as a consequence of a perturbation of the Mtb-immunity. In this regard, it has been shown that COVID-19 induces a frequency decline in Mtb-specific CD4 T-cells in subjects with TB infection, thus potentially impairing the Mtb infection control [17]. However, additional studies are needed to fully understand if these immunological dysregulations may have an impact on the clinical outcome of each disease.

\section{Aim of this review and search methods}

The definition of TB infection has evolved over time, and recent evidence suggests that a "continuum" of situations or stages exists from TB infection to TB disease. This review describes, through the history of definitions, these different stages and their relationships. It also summarises the new developments in prevention, diagnosis and treatment of TB infection as well as their public health and policy implications.

We made a rapid and non-systematic search of the literature using the keywords "TB", "TB infection", "definitions" to identify a minimum set of references from an electronic database (PubMed), existing guidelines on TB infection and grey literature without time limitations.

\section{History of definitions}

Latent tuberculosis infection (LTBI) was the term traditionally used to indicate TB infection. This term has been used to define a state of persistent immune response to stimulation by Mtb antigens through tests such as the tuberculin skin test (TST) or an interferon (IFN)- $\gamma$ release assay (IGRA) without clinically active TB [18]

Recently WHO adopted the term "TB infection" to better indicate "a continuum" in the process, which follows inhalation of bacilli and may lead to clinically manifested TB disease [7, 8].

The following paragraphs educationally describe the stages so far included in the TB spectrum of disease. New insights into TB pathogenesis are also considered $[19,20]$

\section{Latent infection or a "continuum" towards TB disease?}

The term LTBI (still commonly used) was introduced for the first time by the Austrian paediatrician Clemens von Pirquet. He developed the TST, in which the "old tuberculin" isolated by Robert Koch was applied to a superficial abrasion of the skin. Clemens von Pirquet observed that the proportion of positive skin reactions increased with age: for example, they were $\sim 5 \%$ in infants, increasing to $\sim 80 \%$ at the age of 10 years, thus indicating an increased risk of acquiring TB with age [5].

Historically, for research, prevention, diagnostic and treatment purposes a dualistic approach was used, including LTBI and (active) TB disease. This oversimplification of the pathogenesis of TB was useful. However, evidence indicates that the spectrum from LTBI to TB disease is much more complex, including a continuous spectrum of situations [21-23]. These situations are regulated from one side by the metabolic activity of Mtb (dormancy, intermittent replication, active replication) and from the other side by the host innate and acquired immunity (which fights to limit the consequences of TB infection) [24].

With this perspective $\mathrm{WHO}$ has suggested to remove the word "latent" leaving the term "TB infection" only $[7,8]$. The stage referred to as "TB 


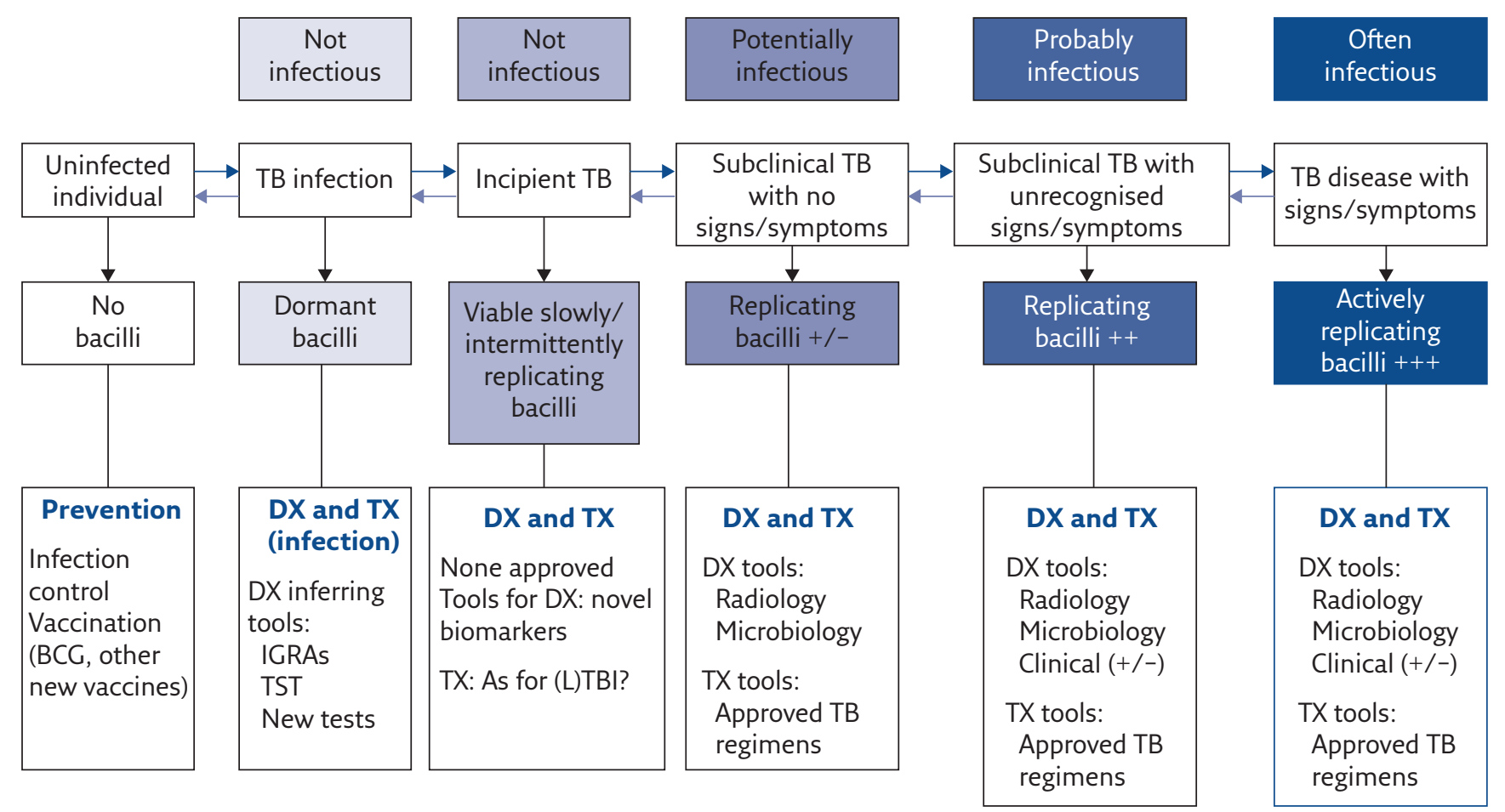

Figure 1 The "continuum process" leading from TB infection to disease. BCG: bacille Calmette-Guérin; DX: diagnosis; TX: treatment; IGRAs: interferon- $\gamma$ release assays; TST: tuberculin skin test; (L)TBI: (latent) TB infection.

infection" includes bacilli at the dormant stage and corresponds to the same term used by WHO. In this article, we describe the evolution of the definition of TB infection, the tools recommended to diagnose and treat it, as well as what is new in terms of the prevention and public health perspectives.

Recently, in an effort to better describe the process (and allowing a future differentiation and tailored clinical approach) two additional clinical stages have been proposed to describe this bi-directional flow in the process going from TB infection to TB diseases: incipient and subclinical TB [24, 25].

To didactically describe the available evidence, in figure 1 we summarise this "continuum" process using six stages: uninfected individual; TB infection; incipient TB; subclinical TB without signs/ symptoms; subclinical TB with unrecognised signs/ symptoms; and TB disease with signs/symptoms.

\section{Incipient TB}

The term incipient TB, which lies between TB infection and subclinical TB, is the most difficult to describe, as the evidence is extremely modest [24]. Bacilli are viable, and probably alternating periods of dormancy (typical of the TB infection state) with periods of slow metabolic activity and replication. The patient is probably not infectious. There are no validated tools to diagnose incipient TB [24]. The infection diagnostic tests (TST and IGRAs) are positive and remain positive in subsequent stages towards TB disease. In future, new biomarkers will enable the specific diagnosis of incipient TB that is going to progress to subclinical or clinical TB, possibly by identifying host ribonucleic acid (RNA) signatures [26-28]. Larger studies are needed to confirm the accuracy of these results.

Considering the low amount of bacilli replication and the minimal lesions present at this stage, today it is still difficult to recommend an "ideal" drug regimen to treat incipient TB $[27,28]$. Future studies are needed to provide insights on the best clinical approach.

\section{Subclinical TB}

Subclinical TB was defined as "disease", due to viable Mtb bacteria, that does not cause clinical TB-related symptoms but causes different abnormalities that can be detected using existing radiological or microbiological assays [24, 25, 29].

It represents an evolution from the stage of TB infection (dormancy, no detectable abnormalities, no tendency to evolve towards the next stages) and incipient TB, with possible intermittent replication of bacilli potentially evolving towards the next stages, without detectable abnormalities [24, 29].

At the subclinical TB stage, the bacilli are replicating, metabolically active, and therefore this stage is potentially infectious. This status can be diagnosed using microbiological and radiological tools. As symptoms can be present or not present (although the patient cannot identify them) the usual clinical criteria are not of much help [29].

Therefore, at the subclinical stage the patients, although potentially infectious, have no symptoms or have minimal ones they cannot 
identify. This finding has several implications. For example, the majority of guidelines recommend to direct patients with chronic cough ( $>2$ or 3 weeks' duration) to bacteriological and radiological screening: in absence of symptoms or capacity to identify them, these patients are not recognised (although they are often infectious). Another example is represented by the inability of screening algorithms based on symptom scores to identify these infectious patients: if radiology is planned only for symptomatic patients, the screening procedure will miss them.

Subclinical TB may have important implications for the spread of the Mtb infection in the community $[4,29]$. Based on these concepts, subclinical TB may represent a very heterogeneous condition, possibly more prevalent than what is expected: using the average prevalence-to-notification ratio of $2: 1$, the prevalence of subclinical TB can be in the order of 7 million, in a total of 14 million patients with TB disease [29].

Evidence of the conditions described above are the results of prevalence surveys where the difference between existing (prevalent) and diagnosed TB cases consisted mainly of culture positive (often sputum smear negative) and symptom-free patients with chest radiological abnormalities [29-32]. Even among sputum smear positive patients with high bacillary load, a proportion of patients ranging from $34 \%$ to $68 \%$ had no symptoms [30].

As summarised in figure 1, the tools available to diagnose subclinical TB are the same as those used to manage "TB disease", although the "clinical" tool may not always be useful, as a proportion of these individuals do not report any signs and/or symptoms. For individuals without symptoms radiological and bacteriological criteria may rely on including a broad category of biomarkers "sensu lato" described below; importantly, to diagnose those with unrecognised symptoms we need to increase the sensitivity threshold of our non-symptomsbased tools [33, 34]. Interesting results indicate that the production and release of Mtb bioaerosols is possible, as reported by the quantitation of $\mathrm{Mtb}$ exhaled during specific respiratory manoeuvres $[33,34]$. Further studies are needed to evaluate the accuracy of this approach to diagnose the different TB stages.

\section{What new tests are available to diagnose TB infection?}

\section{TST}

TB infection is a state with a few bacilli in a dormant stage. In the absence of technologies to detect dormant bacilli, the obvious alternative is to detect or try to determine the host response to the immunogenic antigens of Mtb. The original approach by von Pirquet [5] evolved and a standard
TST was developed based on the purified protein derivative (PPD) RT23 from the Statens Serum Institut in Copenhagen, Denmark. The test was largely used in the 1950s and 1960s to evaluate the annual risk of TB infection (ARTI) and to estimate the incident TB cases expected in a given population [35]. The test was also used clinically to diagnose TB infection: 5 international units (IU) of PPD is intradermally injected into the forearm, and the resulting induration is read in millimetres after 48-72 h.

As it is inexpensive and does not require quality laboratory expertise to administer, the TST became popular and it is still widely used. Details on test requirements, drawbacks and advantages, as well as a head-to-head comparison between TST and IGRA tests are summarised in table 1.

\section{IGRAs and other in vitro tests for TB infection diagnosis}

A crucial step for the development of newer and more specific tests than the TST to diagnose TB infection was the discovery of a specific region of the Mtb DNA (RD1), which was absent from Mycobacterium bovis bacille Calmette-Guérin (BCG), but present in the strains of Mtb complex [36]. This region encodes ESAT- 6 and CFP-10 which are Mtb specific.

Peripheral blood mononuclear cells (PBMCs), in the ex vivo setting, were stimulated with ESAT-6 and CFP-10 peptides or recombinant proteins to induce IFN- $\gamma$ secretion. This Mtb-induced IFN- $\gamma$ response was shown to correlate with the grade of exposure to a TB index case more than TST [37, 38]. This finding prompted the development of assays based on IFN- $\gamma$ release (IGRAs), in which either whole blood or PBMCs are stimulated with RD1encoded antigens.

The two common IGRAs include the QuantiFERON (QFT) system (now QuantiFERONPlus) and the T-SPOT.TB, which are based on the whole blood or PBMC stimulation, respectively, with Mtb-specific peptides. New enzyme-linked immunosorbent assay (ELISA)-based whole blood IGRAs are commercially available. These new tests include Standard E TB-Feron (SD Biosensor, Republic of Korea), AdvanSure TB-IGRA ELISA (LG Chem, Republic of Korea) and LIOFeron TB/LTBI (LIONEX Diagnostics \& Therapeutics $\mathrm{GmbH}$, Braunschweig, Germany) [39]. All these assays are based on IFN- $\gamma$ detection by ELISA after whole blood stimulation in three or four tubes containing recombinant proteins or peptides from Mtb. Interestingly, a new test based on IFN- $\gamma$-induced protein-10 (IP-10) detection by ELISA in whole blood stimulated with Mtb antigen peptides (rBiopharm, Darmstadt, Germany) is now available. This chemokine is expressed at higher levels compared to IFN- $\gamma$ and is associated with TB [10, 40-42].

IGRAs or the assay based on IP-10 detection have several advantages (table 1 ): they do not require a 


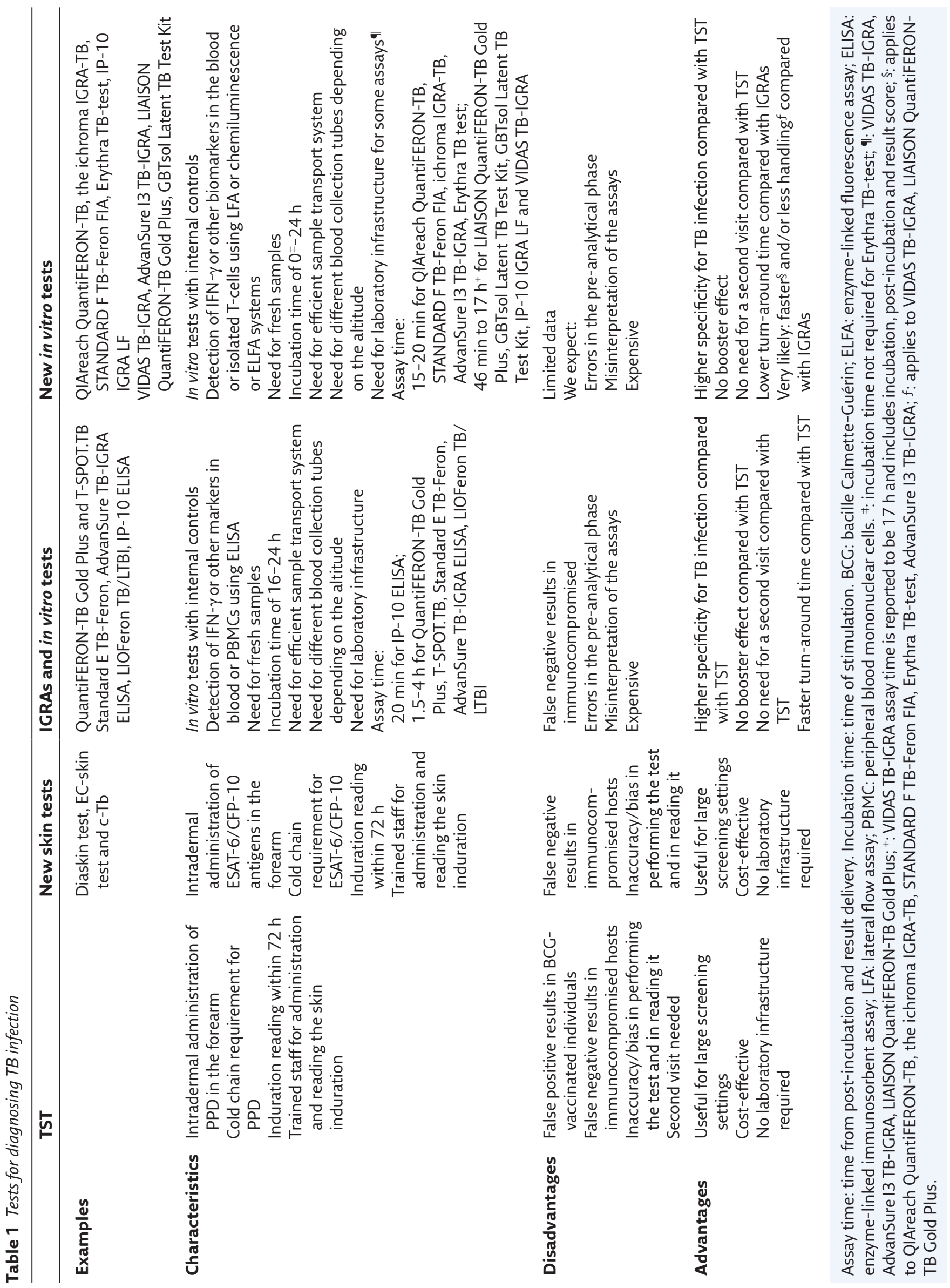


second visit for reading, unlike the TST; have lower false-negative results in immunosuppressed individuals; and do not cause false-positive results in BCG-vaccinated and nontuberculous mycobacteriainfected individuals (due to the specificity of the Mtb antigens used (ESAT-6 and CFP-10)). The results from head-to-head comparisons between IGRAs and TST indicate that the higher cost of IGRAs is an issue.

Recent research, including prospective studies, compared TST against commercially available IGRAs in TB contacts and recent immigrants in low TB-incidence settings. The positive predictive value (PPV) for progression to active TB was evaluated. The PPV of the QuantiFERON-TB Gold In-Tube (a whole blood IGRA) was 3.3\%, while the reported PPV of T-SPOT TB was $4.2 \%$ and that of TST (a $15 \mathrm{~mm}$ cut-off was used to define positivity) was 3.5\% [43].

The existing tests for TB diagnosis and treatment have a low PPV for the identification of subjects progressing to TB disease. Therefore, we need to treat a large number of individuals with a positive IGRA to prevent one individual from developing TB disease. Therefore, the priority is to develop a new generation of tests that predict with higher accuracy those that will progress to disease and focus the diagnostic effort on this target population $[26,28,44,45]$.

\section{Innovative tests for TB infection diagnosis}

Innovative skin tests

Recent new skin tests have been proposed, e.g. the Diaskin test (Generium, Russian Federation), the ESAT6-CFP10 (EC-skin test) (Anhui Zhifei Longcom Biopharmaceutical Co. Ltd, China) and the c-Tb (Serum Institute of India, India) [39]. These tests measure the skin reaction to the intradermal inoculation of ESAT- 6 and CFP-10 Mtb antigens. Therefore, like IGRAs, they are unaffected by a prior BCG vaccination or contacts with environmental mycobacteria [46]. Moreover, their accuracy for the diagnosis of TB infection is similar to that of IGRAs [39, 47, 48]. Like TST, the Diaskin test, EC-skin test and c-Tb tests can be used in largescale screening settings, are cost-effective and do not need the infrastructure of a laboratory. However, as these tests are based on intradermal injection of the antigens, the skin reaction needs to be read after $48-72 \mathrm{~h}$.

Innovative IGRAs and in vitro tests for TB infection diagnosis

A comprehensive landscape of tests for TB infection diagnosis has been recently described [39]. The simplified versions of IGRAs encompasses the lateral flow assays (LFAs). LFAs for TB infection diagnosis include the QIAreach QuantiFERON-TB
(QIAGEN, The Netherlands), the ichroma IGRA-TB (Boditech Med Inc., Republic of Korea) and the STANDARD F TB-Feron FIA (SD Biosensor, Republic of Korea). These tests are based on the IFN- $\gamma$ response detection after whole blood stimulation with ESAT- 6 and CFP-10 antigens (STANDARD F TB-Feron FIA also includes the TB 7.7 antigen) for $16-24 \mathrm{~h}$. All these tests, with the exception of QIAreach QuantiFERON-TB that generates a qualitative result (positive or negative), generate a quantitative result in 15-20 min using a plate reader. Another IFN- $\gamma$-based LFA is the Erythra TB-KIT (Erythra Inc., Stanford, CA, USA), which is a lateral flow chromatography assay based on PPD stimulation of whole blood. Finally, LFA technologies employing IP-10 as readout marker have been developed. This test, called IP-10 IGRA LF (developed by rBiopharm) is based on whole blood stimulation with ESAT- 6 and CFP-10 antigens. Not much information is available about the last two tests.

Other than LFAs, efforts have been made to improve the IGRAs based on the QFT system. New tests such as the VIDAS TB-IGRA (bioMérieux, Marcy-l'Étoile, France), AdvanSure I3 TB-IGRA (LG Chem, Republic of Korea) and LIAISON QuantiFERON-TB Gold Plus (QIAGEN, The Netherlands) have been developed. These tests share with the QFT-Plus system the IFN- $\gamma$ measurement after the stimulation of whole blood with Mtb ESAT- 6 and CFP-10 peptides. The process has been fully automated in the VIDAS TB-IGRA which generates results in $17 \mathrm{~h}$ through an enzymelinked fluorescent assay. Conversely, AdvanSure I3 TB-IGRA and LIAISON QuantiFERON-TB Gold Plus are chemiluminescent assays that have a faster assay time (15-46 min post-incubation) compared with other available ELISA-based tests.

Finally, GBTsol Latent TB Test Kit (Glory Biotechnologies Corp., Republic of Korea) is a new test based on the direct detection of antigen-specific T-cells through the complex MHC-ESAT6 peptides or CFP10 peptides. Both $\mathrm{MHCI}$ and II are included to stimulate CD8 and CD4 T-cells, respectively. The MHC-peptide complexes are detected through a micro-filter separation of whole blood cells. This test provides results in only $1 \mathrm{~h}$.

New perspectives: the microbiological diagnosis of TB infection

While these new skin tests and IGRAs represent an operational advantage compared with existing routine tests [49], these assays are based on the detection of host responses to Mtb and therefore are limited by their inability to distinguish responding but uninfected individuals (in whom infection may have been eliminated by the host response or by preventive therapy) from those who are responding and still harbouring viable infection. Differentiating these states requires detection of the mycobacteria itself, rather than host factors. Despite scepticism, 
it has been recently shown that blood Mtb DNA can be used as a biomarker of TB infection [50] and this is based on the discovery that Mtb DNA could be detected in CD34+ long-term pluripotent haematopoietic stem cells harvested from the peripheral blood of IGRA-positive asymptomatic individuals living in a low-incidence setting [51-53]. PCR has also been used to detect Mtb DNA in the peripheral blood of three out of 18 asymptomatic TB contacts living in a different low-incidence setting of the UK, of whom two individuals progressed to active TB after 7 months [54]. The first longitudinal study to corroborate the robustness of these findings in a high-incidence setting (Ethiopia) found that Mtb DNA was detectable in PBMCs of $79 \%$ of asymptomatic participants, with its presence being more common in HIV-infected versus HIV-uninfected individuals. Administration of preventive therapy to HIV-infected participants reduced prevalence of PCR-detected Mtb DNA from 95\% at baseline to $54 \%$ post-treatment; the first time that a biomarker of TB infection has been shown to be responsive to treatment [50]. Hopefully, these exciting results will be the start of the development of a new generation of TB infection biomarkers based on the detection of bacillary, rather than host factors.

\section{Novel approaches for the detection of host response to Mtb infection}

Several efforts have been made to identify host responses associated with TB infection. For this purpose, different antigen combinations or different read-outs have been evaluated in ex vivo assays. The growing awareness that TB can be considered a spectrum of "disease states" has encouraged the development of tests to identify individuals at high risk of developing active TB disease. Whole blood gene expression signatures have been identified in HIV-uninfected and -infected individuals [26, 28, $44,45]$. These signatures can predict short-term progression to active disease and positron emission tomography-computed tomography images confirmed intrathoracic lesions with increased uptake of radiolabelled glucose in the absence of specific symptoms or chest radiography images indicative of TB disease [45].

\section{What is new for treatment of TB infection?}

Management of TB infection is a core pillar of the WHO End TB Strategy, with the ultimate goal of eliminating TB [7, 8, 55].

The efficacy of the preventive TB regimens presently recommended by $\mathrm{WHO}$ is rather high, ranging from $60 \%$ to $90 \%[7,8]$. WHO emphasises the importance of balancing potential benefits and harms, taking into consideration cost-effectiveness and feasibility. As discussed, no approved routine tests can detect the mycobacteria in TB infection, while existing tests (such as IGRAs and the TST), assess the host-response: so, no test is perfect [7, 8, 24, 29]. Furthermore, testing has an economic cost. Therefore, mass-screening for TB infection is not recommended, while targeted screening of individuals at risk for TB progression would make the approach much more feasible and cost-effective.

There is evidence that shorter regimens (e.g. 3 months of once weekly rifapentine plus isoniazid; 3 months of daily isoniazid plus rifampicin; 1 month of daily isoniazid plus rifapentine) have higher treatment adherence and completion rates compared with those reported with the traditional regimens [7, 8, 55-57].

In the latest guidelines WHO also recommends, in settings with rampant TB transmission, to continue the daily isoniazid regimen for at least 36 months in adults and adolescents living with HIV, given the substantial risk of TB re-infection $[7,8]$.

However, to date, the management of TB patients relies on standard therapy protocols that do not consider the variability of the host or Mtb pathogenicity. Therefore, the introduction of the personalised medicine concepts may allow individual selection of the best therapy regimens [58]. In the past few years, the so-called omics approaches have identified several biomarkers that may be promising for TB disease management. New insights may be provided by next generation sequencing (NGS) technology predicting drugresistance or susceptibility. NGS drug-resistance profiles will anticipate treatment decisions, may be useful to predict treatment failure or risk of relapse related to low response or resistance and will be of utmost worth for multidrug-resistant TB [59]. In parallel, mycobacterial transcriptional profiles or proteomic signature may contribute to evaluate the response to antibiotics or to achieve a better understanding of the mechanisms responsible for drug resistance, thus favouring the development of new drugs. Besides mycobacterial biomarkers, systems biology approaches have identified host biomarkers linked to treatment response. Several genetic factors have been identified; in particular, a Mendelian susceptibility to mycobacterial disease related to genes involved in IFN- $\gamma$ immunity has been described [60]. Moreover, the study of polymorphisms of the leukotriene A4 hydrolase may be useful to identify patients with TB meningitis who can benefit from the addition of dexamethasone therapy [61, 62]. Moreover, it has been demonstrated that hypermethylation of genes involved in the anti-mycobacterial immune response during TB dampens host immune responsiveness even after successful therapy, highlighting the potentials of epigenetics in TB management [63]. Similarly, proteomic and metabolic signatures in blood, sputum or urine could contribute to evaluate treatment response and the disease outcome [59]. All these new 
biomarkers are promising; however, their clinical relevance should be evaluated in large-scale clinical studies [59]. This is particularly important for drug resistant-TB patients needing longer therapy regimens with more toxic drugs and at high risk of treatment failure. Given the evolution of the definition of TB infection and looking into the omics future, the efficacy of TB therapy regimens might be even higher when we will be able to correctly diagnose which individuals to treat.

\section{What is new on TB infection from the public health perspective?}

Given the pivotal role that successful management of TB infection has in pursuing TB elimination [64], there are several important public health implications which deserve to be briefly discussed $[7,8,55,56]$.

\section{Core indicators}

WHO established three main indicators to monitor the implementation of TB infection management strategies $[7,8]$, focusing on the individuals for whom treatment of TB infection is recommended (HIV-coinfected, contacts, patients treated with immunosuppressive drugs (e.g. tumour necrosis factor- $\alpha$ inhibitors) and in dialysis, candidates for transplantation, silicosis patients, prisoners, healthcare workers, migrants, homeless, and drug abusers among others):

1) Contact investigation coverage: percentage of coverage of confirmed TB patients evaluated for TB infection (and disease) out of those eligible;

2) Treatment of TB infection coverage: percentage of individuals initiating treatment for TB infection out of those eligible; and

3) Treatment of TB infection completion: percentage of individuals completing treatment for TB infection out of those eligible.

\section{Monitoring the "cascade of care"}

The best approach to monitor and evaluate if the TB infection management policy works, both at the individual clinic level and from a national surveillance perspective, is to evaluate the so-called "cascade of care" described by Alsdurf et al. [65]. The purpose of the "cascade of care" is to describe the different steps from diagnosis of TB infection to the completion of its treatment. Knowing the proportion of individuals at the different steps of the "cascade" is important.

Ideally, a public health impact is possible if the vast majority of individuals with treatment indications of TB infection are diagnosed and complete their treatment. The description of the "cascade" includes the following steps (in number and percentage, the first step being, by definition, $100 \%$ ): intended for TB infection screening; initially tested; receiving test results; referred if the test is positive; completed medical examinations; recommended for treatment; accepted and started treatment; completed treatment. Importantly, adverse events of any drug regimens should be actively monitored $[7,8]$.

\section{Implementing the register for TB infection}

In order to describe the cascade of care, a register for TB infection is key, in parallel with what is being done for TB disease. Currently, a few countries have already implemented this TB infection register [56], making the collection, analysis and interpretation of data available from different clinics possible.

\section{TB infection and subclinical disease policy development}

Diagnosis and treatment of TB infection are considered the core intervention for TB elimination [29]. Recent WHO guidelines [7, 8] have summarised what is necessary to programmatically organise diagnosis and treatment of TB infection. The capacity to impact groups with a higher probability of developing TB when infected with Mtb (as discussed above, this group includes patients with HIV co-infection, and comorbidities like diabetes, genetic predisposing factors, malnutrition, treatment with glucocorticoids or biological agents, solid organ transplantation, and malignancies among others) will make a difference; this would allow achievement of both an individual-level benefit (for the individuals diagnosed and treated) and the public health goal of TB elimination [55, 56].

We have discussed above the potential relevance of subclinical TB disease towards the global TB burden (tentatively 7 of the 14 million cases) [29]. As summarised in figure 1 , subclinical TB includes individuals with no symptoms or unrecognised symptoms. We have also discussed the possibility that in the absence of cough (historically considered the symptom of TB) transmission of Mtb can occur [29]. While radiological and microbiological tools can identify these cases, symptom-based clinical algorithms usually cannot, as previously discussed, based on the evidence from prevalence surveys.

Subclinical TB had been described as a stage preceding detectable active TB by a couple of weeks or months. This is probably inaccurate as some individuals with subclinical TB never reach the status of TB disease "with symptoms" or spontaneously regress to latent TB (or even to the uninfected stage; figure 1) without being ever diagnosed with TB [29]. This means that subclinical TB may have a major role in transmitting Mtb. 
Table 2 Research priorities for TB infection

\begin{tabular}{|c|c|}
\hline Areas for research & Specific areas \\
\hline $\begin{array}{l}\text { Risks of progression from TB } \\
\text { infection to TB disease }\end{array}$ & $\begin{array}{l}\text { Evaluation of the likelihood of progressing from TB infection to TB disease in the various } \\
\text { at-risk populations } \\
\text { Evaluation of the incidence and risk of progression to TB disease in the following groups } \\
\text { of patients: } \\
\text { Diabetes mellitus } \\
\text { Harmful alcohol use } \\
\text { Tobacco use } \\
\text { Underweight } \\
\text { Silica exposure } \\
\text { Steroid treatment } \\
\text { Rheumatological diseases } \\
\text { Cancer } \\
\text { Evaluation of the harms including stigmatisation and acceptability of testing and TB } \\
\text { preventive therapy in risk groups }\end{array}$ \\
\hline $\begin{array}{l}\text { Improved diagnostic tests and } \\
\text { defining the performance of latent } \\
\text { TB tests in at-risk populations }\end{array}$ & $\begin{array}{l}\text { Tests with improved performance and predictive value for progression to TB disease } \\
\text { Performance of existing latent TB tests in at-risk populations } \\
\text { Tests to determine TB reinfection } \\
\text { How to use existing tests to best determine LTBI }\end{array}$ \\
\hline
\end{tabular}

To adapt existing policies to fully capture the concepts discussed in this article, more evidence is necessary on the exact contribution of transmission from subclinical TB and the role of spontaneous regression (and eventually cure) from one side [29] and of "re-infection" on the other side [66].

Furthermore, more research is needed to understand the role played by new tools (e.g. transcriptomic assays, C-reactive protein) in the new diagnostic algorithms.

If subclinical TB is confirmed to contribute to TB transmission (and the global TB burden), new policies and algorithms focused on subclinical TB are necessary, based on radiological and microbiological tools as well as on new tools beyond symptom-based approaches. Active screening of populations likely to have higher prevalence of subclinical TB (e.g. contacts) will be the next step, pending evidence on its cost-effectiveness.

\section{Priorities for research}

The priorities for research are summarised in table 2. WHO indicates three major areas where research should be focused: evaluating the risks for progression from TB infection to TB disease in at-risk groups; defining the best algorithm to exclude TB disease; and the need for improved tests and the performance of existing TB infection tests in at-risk populations. There is a need to better diagnose TB infection in at-risk populations requiring TB preventive therapy. Furthermore, although not discussed specifically in this review, the best approach to treat TB infection in recent contacts of multidrug-resistant patients is an important research priority.

\section{Conclusions}

This review describes, through the history of definitions, the spectrum from TB infection to TB disease and their clinical and public health implications. As of today, this includes a "continuum" of situations didactically reported in different stages.

While for the majority of the stages there are diagnostic and treatment tools available, incipient TB lies in between TB infection and subclinical TB. Although at the moment we do not have diagnostic and treatment weapons beyond those for TB infection, the future availability of new tools will further enhance the importance of this stage, both from a clinical and public health perspective. To date, the omics approaches have identified promising biomarkers for diagnosis and treatment management. However, future randomised clinical studies are needed to implement systems biology findings in clinical practice and to introduce different recommendations for people at high risk into the "continuum" process to TB disease.

Finally, the importance of subclinical TB and the related relevant burden on Mtb transmission requires attention from both clinicians and public health experts. 


\section{Self-evaluation questions}

Select one correct answer for each question.

1. How many stages can be used to didactically describe the process from TB infection to disease?

a) Two main stages, latent TB infection and TB disease

b) Six stages, including uninfected individual, TB infection, incipient TB, subclinical TB without signs/symptoms, subclinical

TB with unrecognised signs/symptoms, and TB disease with signs/symptoms

c) The process is a "continuum" and cannot be described by means of specific stages

d) Two main stages, TB infection and TB disease

2. What are the WHO-recommended tests to diagnose TB infection?
a) TST and IGRAs
b) The QuantiFERON-TB and T-SPOT TB tests in low TB-incidence countries
c) TST in resource-limited settings
d) TST and IGRAs in the absence of clinically active TB

3. What is the efficacy of the WHO-recommended regimens to treat TB infection?

a) The efficacy of the regimens presently recommended by WHO is very high and $>90 \%$

b) The efficacy of the regimens presently recommended by $\mathrm{WHO}$ is high and $>80 \%$

c) The efficacy of the regimens presently recommended by WHO is not yet very high, being still $<50 \%$

d) The efficacy of the regimens presently recommended by WHO is rather high, ranging from $60 \%$ to $90 \%$

4. What is the "cascade of TB infection care"?

a) The best approach to monitor and evaluate how in a given setting the management of TB infection is conducted: how many of the individuals intended for TB infection screening are able to complete the prescribed regimen

b) The proportion of patients who interrupt treatment because of adverse events of the regimen

c) The \% decline of individuals interrupting treatment at the different steps of the process

d) The proportion of patients who start treatment of TB infection

\section{Key points}

- Management of TB infection is the core element of the TB elimination strategy.

- Historically, for research, prevention, diagnostic and treatment purposes a dualistic approach was used, including LTBI and (active) TB disease.

- To didactically describe the "continuum process" leading from TB infection to disease, six stages are described: uninfected individual; TB infection; incipient TB; subclinical TB without signs/symptoms; subclinical TB with unrecognised signs/symptoms; and TB disease with signs/symptoms.

- $\quad$ The tests recommended by WHO to diagnose TB infection and the regimens recommended to treat it are summarised in this review.

- Finally, the policy and research implications of the new WHO recommendations on TB infection management are described. 


\section{Affiliations}

Giovanni Battista Migliori' ${ }^{1}$ Catherine W.M. Ong ${ }^{2,3}$, Linda Petrone ${ }^{4}$, Lia D’Ambrosio ${ }^{5}$, Rosella Centis ${ }^{1}$, Delia Goletti ${ }^{4}$

'Servizio di Epidemiologia Clinica delle Malattie Respiratorie, Istituti Clinici Scientifici Maugeri IRCCS, Tradate, Italy. ${ }^{2}$ Dept of Medicine, Yong Loo Lin School of Medicine, National University of Singapore, Singapore. ${ }^{3}$ Institute for Health Innovation and Technology (iHealthtech), National University of Singapore, Singapore. ${ }^{4}$ Translational Research Unit, National Institute for Infectious Diseases "Lazzaro Spallanzani”-IRCCS, Rome, Italy. ${ }^{5}$ Public Health Consulting Group, Lugano, Switzerland.

Suggested answers

1. b.

2.d.

3. d.

4. a.

\section{Acknowledgements}

The article is part of the activities of the Global Tuberculosis Network (GTN) and of the WHO Collaborating Centre for Tuberculosis and Lung Diseases, Tradate (ITA-80, 2017-2020-GBM/RC/LDA); we also thank the support from the Italian Ministry of Health (Ricerca Corrente, Linea 4).

\section{Conflict of interest}

D. Goletti reports grants (to her institution) and consulting fees from Biomerieux and consulting fees and payment or honoraria for a lecture from Qiagen, outside the submitted work. G.B. Migliori, C.W.M. Ong, L. Petrone, L. D’Ambrosio and R. Centis have nothing to disclose.

\section{References}

1. Cohen A, Mathiasen VD, Schön T, et al. The global prevalence of latent tuberculosis: a systematic review and meta-analysis. Eur Respir J 2019; 54: 1900655.

2. Loddenkemper R, Murray JF. History of tuberculosis. In: Migliori GB, Raviglione MC, eds. Essential Tuberculosis. Cham, Springer Nature Switzerland AG, 2021; pp. 6-9.

3. Tagliani E, Nikolayevskyy V, Tortoli E, et al. Laboratory diagnosis of tuberculosis. In: Migliori GB, Bothamley G, Duarte R, Rendon A, eds. Tuberculosis (ERS Monograph). Sheffield, European Respiratory Society, 2018; pp. 99-115.

4. Migliori GB, Nardell E, Yedilbayev A, et al. Reducing tuberculosis transmission: a consensus document from the World Health Organization Regional Office for Europe. Eur Respir J 2019; 53: 1900391.

5. Goletti D, Martineau AR. Pathogenesis and immunology of tuberculosis. In: Migliori GB, Raviglione MC, eds. Essential Tuberculosis. Cham, Springer Nature Switzerland AG, 2021; pp. 20-28.

6. Barreira-Silva P, Torrado E, Nebenzahl-Guimaraes $\mathrm{H}$, et al. Aetiopathogenesis, immunology and microbiology of tuberculosis. In: Migliori GB, Bothamley G, Duarte R, Rendon $A$, eds. Tuberculosis (ERS Monograph). Sheffield, European Respiratory Society, 2018; pp. 62-82.

7. World Health Organization. WHO consolidated guidelines on tuberculosis. Module 1: prevention - tuberculosis preventive treatment. Geneva, World Health Organization, 2020. https://apps.who.int/iris/bitstream/han dle/10665/331170/9789240001503-eng.pdf

8. World Health Organization. WHO operational handbook on tuberculosis. Module 1: prevention - tuberculosis preventive treatment. Geneva, World Health Organization, 2020. https://apps.who.int/iris/bitstream/han dle/10665/331525/9789240002906-eng.pdf

9. Goletti D, Petrone L, Ippolito G, et al. Preventive therapy for tuberculosis in rheumatological patients undergoing therapy with biological drugs. Expert Rev Anti Infect Ther 2018; 16: 501-512.

10. Petrone L, Cannas A, Aloi F, et al. Blood or urine IP-10 cannot discriminate between active tuberculosis and respiratory diseases different from tuberculosis in children. Biomed Res Int 2015; 2015: 589471.

11. Ong CWM, Migliori GB, Raviglione M, et al. Epidemic and pandemic viral infections: impact on tuberculosis and the lung. Eur RespirJ 2020; 56: 2001727.
12. Boulle A, Davies MA, Hussey $H$, et al. Risk factors for coronavirus disease 2019 (COVID-19) death in a population cohort study from the Western Cape Province, South Africa. Clin Infect Dis 2020; in press [https://doi.org/10.1093/cid/ ciaa1198].

13. Faqihi F, Alharthy A, Noor A, et al. COVID-19 in a patient with active tuberculosis: a rare case-report. Respir Med Case Rep 2020; 31: 101146

14. Motta I, Centis R, D’Ambrosio L, et al. Tuberculosis, COVID19 and migrants: preliminary analysis of deaths occurring in 69 patients from two cohorts. Pulmonology 2020; 26: 233-240.

15. Musso M, Di Gennaro F, Gualano G, et al. Concurrent cavitary pulmonary tuberculosis and COVID-19 pneumonia with in vitro immune cell anergy. Infection 2021; in press [https:// doi.org/10.1007/s15010-021-01576-y].

16. Davies MA. HIV and risk of COVID-19 death: a population cohort study from the Western Cape Province, South Africa. medRxiv 2020; preprint [https://doi.org/10.1101/2020.07. 02.20145185].

17. Riou C, Du Bruyn E, Stek C, et al. Relationship of SARS-CoV-2specific CD4 response to COVID-19 severity and impact of HIV-1 and tuberculosis co-infection. J Clin Invest 2021; 131: e149125.

18. Getahun H, Matteelli A, Abubakar I, et al. Management of latent Mycobacterium tuberculosis infection: WHO guidelines for low tuberculosis burden countries. Eur RespirJ 2015; 46: 1563-1576.

19. Migliori GB, Visca D, van den Boom M, et al. Tuberculosis, COVID-19 and hospital admission: consensus on pros and cons based on a review of the evidence. Pulmonology 2021; 27: 248-256.

20. Tadolini M, Codecasa LR, García-García JM, et al. Active tuberculosis, sequelae and COVID-19 co-infection: first cohort of 49 cases. Eur Respir J 2020; 56: 2001398.

21. Barry CE 3rd, Boshoff HI, Dartois V, et al. The spectrum of latent tuberculosis: rethinking the biology and intervention strategies. Nat Rev Microbiol 2009; 7: 845-855.

22. Lewinsohn DM, Lewinsohn DA. New concepts in tuberculosis host defense. Clin Chest Med 2019; 40: 703-719.

23. Delogu G, Goletti D. The spectrum of tuberculosis infection: new perspectives in the era of biologics. J Rheumatol Suppl 2014; 91: 11-16.

24. Drain PK, Bajema KL, Dowdy D, et al. Incipient and subclinical tuberculosis: a clinical review of early stages and progression of infection. Clin Microbiol Rev 2018; 31: e00021-18. 
25. Esmail H, Barry CE 3rd, Young DB, et al. The ongoing challenge of latent tuberculosis. Philos Trans R Soc Lond B Biol Sci 2014; 369: 20130437.

26. Esmail H, Cobelens F, Goletti D. Transcriptional biomarkers for predicting development of tuberculosis: progress and clinical considerations. Eur Respir J 2020; 55: 1901957.

27. Mendelsohn SC, Fiore-Gartland A, Penn-Nicholson A, et al. Validation of a host blood transcriptomic biomarker for pulmonary tuberculosis in people living with HIV: a prospective diagnostic and prognostic accuracy study. Lancet Glob Health 2021; 9: e841-e853.

28. Scriba TJ, Fiore-Gartland A, Penn-Nicholson A, et al. Biomarker-guided tuberculosis preventive therapy (CORTIS): a randomised controlled trial. Lancet Infect Dis 2021; 21 354-365

29. Kendall EA, Shrestha S, Dowdy DW. Reply to: subclinical tuberculosis: some flies in the ointment. Am J Respir Crit Care Med 2021; 203: 1328-1329.

30. Onozaki I, Law I, Sismanidis C, et al. National tuberculosis prevalence surveys in Asia, 1990-2012: an overview of results and lessons learned. Trop Med Int Health 2015; 20 $1128-1145$.

31. van't Hoog AH, Meme HK, Laserson KF, et al. Screening strategies for tuberculosis prevalence surveys: the value of chest radiography and symptoms. PLoS One 2012; 7: e38691.

32. Frascella B, Richards AS, Sossen B, et al. Subclinical tuberculosis disease - a review and analysis of prevalence surveys to inform definitions, burden, associations, and screening methodology. Clin Infect Dis 2021; 73: e830-e841.

33. Patterson B, Wood R. Is cough really necessary for TB transmission? Tuberculosis (Edinb) 2019; 117: 31-35.

34. Esmail H, Dodd PJ, Houben RMGJ. Tuberculosis transmission during the subclinical period: could unrelated cough play a part? Lancet Respir Med 2018; 6: 244-246.

35. Styblo K. The relationship between the risk of tuberculous infection and the risk of developing infectious tuberculosis Bull Int Union Tuberc Lung Dis 1985; 60: 117-119.

36. Goletti D, Lee MR, Wang JY, et al. Update on tuberculosis biomarkers: from correlates of risk, to correlates of active disease and of cure from disease. Respirology 2018; 23 455-466.

37. Barcellini L, Borroni E, Brown J, et al. First evaluation of QuantiFERON-TB Gold Plus performance in contact screening. Eur Respir J 2016; 48: 1411-1419.

38. Ewer K, Deeks J, Alvarez L, et al. Comparison of T-cell-based assay with tuberculin skin test for diagnosis of Mycobacterium tuberculosis infection in a school tuberculosis outbreak. Lancet 2003: 361: 1168-1173.

39. Hamada Y, den Boon S, Cirillo DM, et al. Framework for the evaluation of new tests for tuberculosis infection. Eur Respir J 2021; 58: 2004078

40. Azzurri A, Sow OY, Amedei A, et al. IFN-gamma-inducible protein 10 and pentraxin 3 plasma levels are tools for monitoring inflammation and disease activity in Mycobacterium tuberculosis infection. Microbes Infect 2005; 7: 1-8.

41. Petrone L, Cannas A, Vanini V, et al. Blood and urine inducible protein 10 as potential markers of disease activity. Int J Tuberc Lung Dis 2016; 20: 1554-1561.

42. Petrone L, Vanini V, Chiacchio T, et al. Evaluation of IP-10 in Quantiferon-Plus as biomarker for the diagnosis of latent tuberculosis infection. Tuberculosis (Edinb) 2018; 111: 147-153.

43. Abubakar I, Drobniewski F, Southern J, et al. Prognostic value of interferon- $\gamma$ release assays and tuberculin skin test in predicting the development of active tuberculosis (UK PREDICT TB): a prospective cohort study. Lancet Infect Dis 2018; 18: 1077-1087.

44. Zak DE, Penn-Nicholson A, Scriba TJ, et al. A blood RNA signature for tuberculosis disease risk: a prospective cohort study. Lancet 2016; 387: 2312-2322.

45. Esmail H, Lai RP, Lesosky M, et al. Characterization of progressive HIV-associated tuberculosis using 2-deoxy-2[(18)F]fluoro-D-glucose positron emission and computed tomography. Nat Med 2016; 22: 1090-1093.
46. Goletti D, Sanduzzi A, Delogu G. Performance of the tuberculin skin test and interferon- $\gamma$ release assays: an update on the accuracy, cutoff stratification, and new potential immunebased approaches. J Rheumatol Supp/ 2014; 91: 24-31.

47. Nikitina IY, Karpina NL, Kasimceva OV, et al. Comparative performance of QuantiFERON-TB Gold versus skin test with tuberculosis recombinant allergen (Diaskintest) among patients with suspected pulmonary tuberculosis in Russia. Int J Infect Dis 2019; 86: 18-24.

48. Ruhwald M, Aggerbeck H, Gallardo RV, et al. Safety and efficacy of the C-Tb skin test to diagnose Mycobacterium tuberculosis infection, compared with an interferon $\gamma$ release assay and the tuberculin skin test: a phase 3, double-blind, randomised, controlled trial. Lancet Respir Med 2017; 5: 259-268.

49. World Health Organization. Framework for the evaluation of new tests for tuberculosis infection. Geneva, World Health Organization, 2020. Available from: https://www.who.int/ publications/i/item/9789240007185

50. Belay M, Tulu B, Younis S, et al. Detection of Mycobacterium tuberculosis complex DNA in CD34-positive peripheral blood mononuclear cells of asymptomatic tuberculosis contacts: an observational study. Lancet Microbe 2021; 2: e267-e275.

51. Bender JG, Unverzagt KL, Walker DE, et al. Identification and comparison of CD34-positive cells and their subpopulations from normal peripheral blood and bone marrow using multicolor flow cytometry. Blood 1991; 77: 2591-2596.

52. Reece ST, Vogelzang A, Tornack J, et al. Mycobacterium tuberculosis-infected hematopoietic stem and progenitor cells unable to express inducible nitric oxide synthase propagate tuberculosis in mice. J Infect Dis 2018; 217: 1667-1671.

53. Tornack J, Reece ST, Bauer WM, et al. Human and mouse hematopoietic stem cells are a depot for dormant Mycobacterium tuberculosis. PLoS One 2017; 12: e0169119.

54. Verma R, Swift BMC, Handley-Hartill W, et al. A novel, highsensitivity, bacteriophage-based assay identifies low-level Mycobacterium tuberculosis bacteremia in immunocompetent patients with active and incipient tuberculosis. Clin Infect Dis 2020; 70: 933-936

55. Matteelli A, Rendon A, Tiberi S, et al. Tuberculosis elimination: where are we now? Eur Respir Rev 2018; 27: 180035.

56. WHO Regional Office for Europe. Tuberculosis elimination in the WHO European Region: review of key actions, with a special focus on tuberculosis infection management. Copenhagen, WHO Regional Office for Europe, 2020.

57. ClinicalTrials.gov. https://clinicaltrials.gov/

58. Lange C, Aarnoutse R, Chesov D, et al. Perspective for precision medicine for tuberculosis. Front Immunol 2020; 11: 566608 .

59. Kontsevaya I, Lange C, Comella-Del-Barrio P, et al. Perspectives for systems biology in the management of tuberculosis. Eur Respir Rev 2021; 30: 200377.

60. Bustamante J, Boisson-Dupuis S, Abel L, et al. Mendelian susceptibility to mycobacterial disease: genetic, immunological, and clinical features of inborn errors of IFN- $\gamma$ immunity. Semin Immunol 2014; 26: 454-470.

61. Tobin DM, Roca FJ, Oh SF, et al. Host genotype-specific therapies can optimize the inflammatory response to mycobacterial infections. Cell 2012; 148: 434-446.

62. Lalvani A, Behr MA, Sridhar S. Innate immunity to TB: a druggable balancing act. Cell 2012; 148: 389-391.

63. DiNardo AR, Rajapakshe K, Nishiguchi T, et al. DNA hypermethylation during tuberculosis dampens host immune responsiveness. J Clin Invest 2020; 130: 3113-3123.

64. Lönnroth K, Migliori GB, Abubakar l, et al. Towards tuberculosis elimination: an action framework for low-incidence countries. Eur RespirJ 2015; 45: 928-952.

65. Alsdurf H, Hill PC, Matteelli A, et al. The cascade of care in diagnosis and treatment of latent tuberculosis infection: a systematic review and meta-analysis. Lancet Infect Dis 2016; 16: $1269-1278$

66. Migliori GB. Global tuberculosis network (GTN). Evolution of programmatic definitions used in tuberculosis prevention and care. Clin Infect Dis 2019; 68: 1787-1789. 